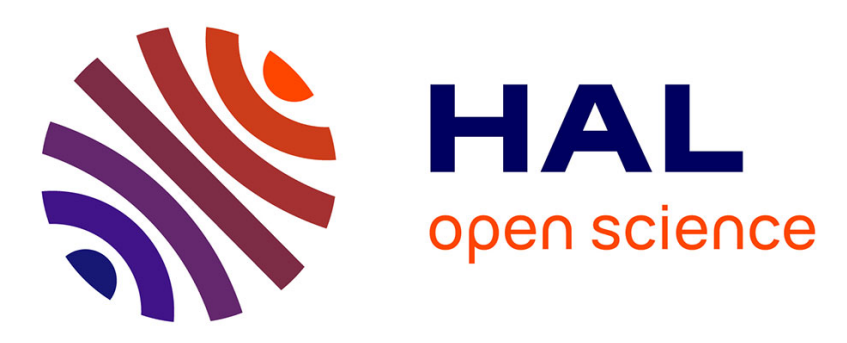

\title{
A refined IV method for closed-loop system identification
}

Marion Gilson, Hugues Garnier, Peter J.W. Young, Paul van den Hof

\section{To cite this version:}

Marion Gilson, Hugues Garnier, Peter J.W. Young, Paul van den Hof. A refined IV method for closedloop system identification. 14th IFAC Symposium on System Identification, SYSID'2006, Mar 2006, Newcastle, Australia. pp.903-908. hal-00089689

\section{HAL Id: hal-00089689 https://hal.science/hal-00089689}

Submitted on 22 Aug 2006

HAL is a multi-disciplinary open access archive for the deposit and dissemination of scientific research documents, whether they are published or not. The documents may come from teaching and research institutions in France or abroad, or from public or private research centers.
L'archive ouverte pluridisciplinaire HAL, est destinée au dépôt et à la diffusion de documents scientifiques de niveau recherche, publiés ou non, émanant des établissements d'enseignement et de recherche français ou étrangers, des laboratoires publics ou privés. 


\title{
A REFINED IV METHOD FOR CLOSED-LOOP SYSTEM IDENTIFICATION
}

\author{
Marion Gilson*, Hugues Garnier*, Peter Young** \\ Paul Van den Hof ${ }^{* * *}$

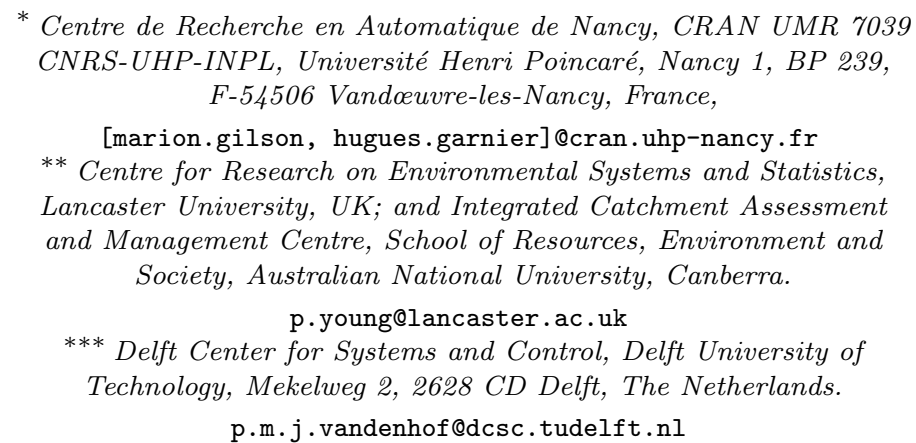

\begin{abstract}
This paper describes an optimal instrumental variable method for identifying discrete-time transfer function models of the Box-Jenkins transfer function form in the closed-loop situation. This method is based on the Refined Instrumental Variable (RIV) algorithm which, because of an appropriate choice of particular design variables, achieves minimum variance estimation of the model parameters. The Box-Jenkins model is the most natural since it does not constrain the process and the noise models to have common polynomials. The performance of the proposed approach is evaluated by Monte Carlo analysis in comparison with other alternative closed loop estimation methods. Copyright (C)2006 IFAC
\end{abstract}

Keywords: System identification; closed-loop identification; optimal instrumental variable.

\section{INTRODUCTION}

For many industrial production processes, safety and economic restrictions are often strong reasons for not allowing identification experiments in open-loop. In such situations, experimental data can only be obtained under closed-loop conditions. The main difficulty in closed-loop identification is due to the correlation between the disturbances and the control signal induced by the loop. Several alternatives are available to cope with this problem (Ljung, 1999). Some particular versions of these methods have been developed more recently in the area of control-relevant identifica- tion. An overview of these recent developments can be found in Van den Hof (1998) and Forssell and Ljung (1999). This paper focuses more specially on instrumental variable techniques which present the great advantage of being able to consistently identify closed-loop plant models while relying on simple linear (regression-like) algorithms.

For closed-loop identification, a basic IV estimator was first suggested by (Young, 1970), assuming knowledge of the controller; and the topic was later discussed in more detail by (Söderström et al., 1987). More recently a so-called 'tailor-made IV algorithm' was proposed (Gilson and Van den 
Hof, 2001), where the closed-loop plant is parameterized using (open-loop) plant parameters. Then, an optimal variance result has been developed in the closed-loop extended IV identification case, revealing consequences for the choice of weights, filters and instruments (Gilson and Van den Hof, 2005). Two bootstrap techniques have been proposed: the first one aims at estimating an ARX model; and the second improves on this by utilizing a more accurate noise model with an ARARX structure. However, both methods still rely on a noise model having the same dynamics as the process model.

In this paper, we propose a new technique which is based on the identification of a more realistic BoxJenkins (BJ) model where the process and the noise models are not constrained to have common polynomials. An apparent problem with this type of BJ model is that simple IV estimation cannot be used directly because the model is non-linearin-the-parameters. However, more sophisticated IV estimation overcomes this limitation. In particular, this paper proposes a solution based on the use of the iterative, optimal Refined Instrumental Variable (RIV) method of estimation (Young, 1976, 1984).

The paper is organized as follows. After the preliminaries, the lower bound of the covariance matrix in case of closed-loop identification is recalled in Section 3. Section 4 shows how the refined IV method provides an estimation approach to the closed loop estimation problem. Finally, in Section 6 , the comparison between different IV methods is illustrated with the help of Monte Carlo simulation examples, demonstrating the efficacy of the proposed approach.

\section{PRELIMINARIES}

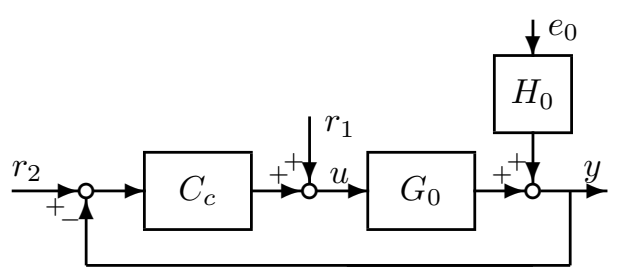

Fig. 1. Closed-loop configuration.

Consider a linear SISO closed-loop system shown in Figure 1. The process is denoted by $G_{0}(z)$ and the controller by $C_{c}(z) ; u(t)$ describes the process input signal, $y(t)$ the process output signal and $e_{0}(t)$ is a sequence of independent identically disturbed random variables of variance $\lambda_{0}$. The external signals $r_{1}(t), r_{2}(t)$ are assumed to be uncorrelated with $e_{0}(t)$. For ease of notation, we also introduce the signal $r(t)=r_{1}(t)+C_{c}(q) r_{2}(t)$. With this notation, the data generating system becomes

$$
\mathcal{S}:\left\{\begin{array}{l}
y(t)=G_{0}(q) u(t)+H_{0}(q) e_{0}(t) \\
u(t)=r(t)-C_{c}(q) y(t)
\end{array}\right.
$$

The real plant $G_{0}$ is considered to satisfy $G_{0}(q)=$ $B_{0}\left(q^{-1}\right) / A_{0}\left(q^{-1}\right)$, while in these expressions $q^{-1}$ is the delay operator, and the numerator and denominator degree is $n_{0}$.

A parameterized process model is considered

$$
\mathcal{G}: G(q, \theta)=\frac{B\left(q^{-1}, \theta\right)}{A\left(q^{-1}, \theta\right)}=\frac{b_{1} q^{-1}+\cdots+b_{n} q^{-n}}{1+a_{1} q^{-1}+\cdots+a_{n} q^{-n}},
$$

and the process model parameters are stacked columnwise in the parameter vector

$$
\theta=\left[\begin{array}{llllll}
a_{1} & \cdots & a_{n} & b_{1} & \cdots & b_{n}
\end{array}\right]^{T} \in \mathbb{R}^{2 n} .
$$

Furthermore, let us denote the open-loop regressor by $\varphi(t) \in \mathbb{R}^{2 n}$, defined as

$$
\begin{aligned}
& \varphi^{T}(t)= \\
& {[-y(t-1) \cdots-y(t-n) u(t-1) \cdots u(t-n)]}
\end{aligned}
$$

If $n=n_{0}$, i.e. the plant $G_{0}$ is contained in the chosen model set, $y(t)$ can be written as

$$
y(t)=\varphi^{T}(t) \theta_{0}+v_{0}(t)
$$

where $\theta_{0}$ denotes the true parameters and $v_{0}(t)=$ $A_{0}\left(q^{-1}\right) H_{0}(q) e_{0}(t)$. Additionally we use the following notation for filtered data

$$
\varphi_{f}(t)=L(q) \varphi(t)
$$

where $L(q)$ is a stable prefilter.

The objective is to estimate $\theta_{0}$ while using the collected data $y(t), u(t)$ and $r(t)$ with or without knowledge of the controller.

Extended-IV. The well-known extended-IV estimate is given by (Söderström and Stoica, 1983)

$$
\begin{aligned}
\hat{\theta}_{x i v}(N)= & \arg \min _{\theta} \|\left[\frac{1}{N} \sum_{t=1}^{N} z_{f}(t) L(q) \varphi^{T}(t)\right] \theta \\
& -\left[\frac{1}{N} \sum_{t=1}^{N} z_{f}(t) L(q) y(t)\right] \|_{Q}^{2},
\end{aligned}
$$

where $z_{f}(t)$ is the instrument vector and $\|x\|_{Q}^{2}=$ $x^{T} Q x$, with $Q$ a positive definite weighting matrix.

By definition, the extended-IV estimate provides a consistent estimate under the following two conditions $^{1}$

- $\overline{\mathbb{E}} z_{f}(t) L(q) \varphi^{T}(t)$ is non singular,

- $\overline{\mathbb{E}} z_{f}(t) L(q) v_{0}(t)=0$.

\footnotetext{
1 The notation $\overline{\mathbb{E}}[]=.\lim _{N \rightarrow \infty} \frac{1}{N} \sum_{t=0}^{N-1} \mathbb{E}[$.$] is adopted$ from the prediction error framework of Ljung (1999)
} 


\section{LOWER BOUND FOR AN IV METHOD}

The choice of the instrumental variable vector $z_{f}(t)$, the number of instruments $n_{z}$, the weighting matrix $Q$ and the prefilter $L(q)$ may have a considerable effect on the covariance matrix $P_{x i v}$ produced by the IV estimation algorithm. In the open-loop situation the lower bound of $P_{x i v}$ for any unbiased identification method is given by the Cramer-Rao bound, which is specified in e.g. (Söderström and Stoica, 1983) and (Ljung, 1999). The closed-loop situation has been investigated recently in Gilson and Van den Hof (2005), so only the main results are recalled here. It has been shown that a minimum value of the covariance matrix $P_{x i v}$ as a function of the design variables $z_{f}(t), L(q)$ and $Q$ exists under the restriction that $z_{f}(t)$ is a function of the external signal $r(t)$ only:

$$
P_{x i v} \geq P_{x i v}^{o p t}
$$

with

$$
P_{x i v}^{o p t}=\lambda_{0}\left[\overline{\mathbb{E}} \tilde{\varphi}_{f}(t) \tilde{\varphi}_{f}^{T}(t)\right]^{-1},
$$

$\tilde{\varphi}_{f}(t)=L(q) \tilde{\varphi}(t)$ and $\tilde{\varphi}(t)$ is the noise-free part of $\varphi(t)$.

It is shown that the minimum variance result can be achieved by the following choice of design variables (see Gilson and Van den Hof (2005) for further explanations):

$$
\begin{aligned}
& Q=I \text { and } n_{z}=2 n, \\
& L(q)=\frac{1}{H_{0}(q) A_{0}\left(q^{-1}\right)}, \\
& z_{f}(t)=L(q) \tilde{\varphi}(t) .
\end{aligned}
$$

Using equations (7) and (8)-(10), the optimal IV estimate is given by

$$
\hat{\theta}_{o p t}(N)=\hat{R}_{z_{f} \varphi_{f}}^{-1}(N) \hat{R}_{z_{f} y_{f}}(N)
$$

with $\hat{R}_{z_{f} \varphi_{f}}(N)=1 / N \sum_{t=1}^{N} z_{f}(t) \varphi_{f}^{T}(t)$, $\hat{R}_{z_{f} y_{f}}(N)=1 / N \sum_{t=1}^{N} z_{f}(t) y_{f}(t)$; and where both the open-loop regressor $\varphi_{f}(t)=L(q) \varphi(t)$ and the output $y_{f}(t)=L(q) y(t)$ are filtered by $L(q)(9)$.

Note that the optimal IV estimator can only be obtained if the true noise model $A_{0}\left(q^{-1}\right) H_{0}(q)^{2}$ is exactly known and therefore optimal accuracy cannot be achieved in practice.

\section{REFINED INSTRUMENTAL VARIABLE ESTIMATION IN THE CLOSED LOOP (RIVCL)}

Based on the results presented in the previous section, it is necessary to take care so that

\footnotetext{
2 Although $A_{0} H_{0}$ is referred to as "noise model" it also involves knowledge of $G_{0}$ through its denominator $A_{0}$.
}

- a model of $A_{0} H_{0}$ is available in order to construct the prefilter $L(q)$ and the instruments $z_{f}(t)$

- the noise free part of the regressor $\tilde{\varphi}(t)$ is needed.

In this connection, while the choice of the instruments and prefilter in the IV method affects the asymptotic variance of the parameter estimates, the consistency properties are generically secured. This suggests that minor deviations from the optimal value (which is not available in practice) will only cause second-order effects in the resulting accuracy. Therefore it is considered sufficient to use consistent, but not necessarily efficient estimates of the dynamics and of the noise when constituting the instrument and the prefilter (Ljung, 1999). In addition, when obtaining the necessary preliminary models, estimation is restricted to linear regression estimates in order to keep the computational procedures simple and tractable. Several attempts have been proposed to handle this problem in closed-loop (see Gilson and Van den Hof (2005) for more details). These previous solutions have used bootstrap techniques since this alternates between computing the vector parameter and forming the new regressor and instruments.

As the optimal IV estimation only requires verification of the assumption $G_{0} \in \mathcal{G}$ (and not $\mathcal{S} \in \mathcal{M}$ ), the first possibility consists of estimating a simple ARX model without any specific noise model identification:

$$
\mathcal{G}: A\left(q^{-1}\right) y(t)=B\left(q^{-1}\right) u(t)+e(t)
$$

Although the results are consistent, it is worth while considering improved noise model estimation in order to construct a more accurate estimate (closer to (11)). One improvement is to use the following ARARX structure (Gilson and Van den Hof, 2005)

$$
A\left(q^{-1}\right) y(t)=B\left(q^{-1}\right) u(t)+\frac{1}{D\left(q^{-1}\right)} e(t)
$$

However, this approach still relies on a noise model having the same dynamics as the process model. A superior alternative is to consider a closed loop BJ Transfer Function (TF) model defined as

$$
\begin{aligned}
\mathcal{G}: y(t) & =\frac{B\left(q^{-1}\right)}{F\left(q^{-1}\right)} u(t)+\frac{C\left(q^{-1}\right)}{D\left(q^{-1}\right)} e(t) \\
\text { and } u(t) & =r(t)-C_{c}(q) y(t) .
\end{aligned}
$$

For most practical purposes, this model is the most natural since it does not constrain the process and the noise models to have common polynomials. The problem introduced by considering (12), however, is that the model is non-linear-in-the-parameters so that simple IV estimation cannot be directly applied. 
Fortunately, this problem of nonlinear estimation can be overcome by utilizing the iterative Refined Instrumental Variable (RIV) method (see Young, $1976,1984)$ that is available in the CAPTAIN Toolbox for Matlab ${ }^{3}$. The simplest explanation of this method is to consider the usual Prediction Error Minimization (PEM) approach within an Optimal Generalized Equation Error setting (Young, 1985a). Here, a suitable error function $\varepsilon(t)$ is given by

$$
\varepsilon(t)=\frac{D\left(q^{-1}\right)}{C\left(q^{-1}\right)}\left[y(t)-\frac{B\left(q^{-1}\right)}{F\left(q^{-1}\right)} u(t)\right] .
$$

This error function is clearly nonlinear in the parameters of the unknown polynomials. However it can be written alternatively as

$$
\varepsilon(t)=\frac{D\left(q^{-1}\right)}{C\left(q^{-1}\right) F\left(q^{-1}\right)}\left[F\left(q^{-1}\right) y(t)-B\left(q^{-1}\right) u(t)\right],
$$

or

$$
\varepsilon(t)=F\left(q^{-1}\right) y_{f}(t)-B\left(q^{-1}\right) u_{f}(t),
$$

where

$$
\left\{\begin{array}{l}
y_{f}(t)=L(q) y(t) \\
u_{f}(t)=L(q) u(t),
\end{array} \quad \text { with } L(q)=\frac{D\left(q^{-1}\right)}{C\left(q^{-1}\right) F\left(q^{-1}\right)} .\right.
$$

Equation (15) is now linear-in-the-parameters of the transfer function model, so that IV methods could be used to estimate the parameters if it were possible to perform the prefiltering operations in (16). In practice, of course, the parameters of the filter $L(q)(16)$ are unknown a priori and so the prefiltering operations must be made adaptive with the iterative RIV algorithm.

\section{THE ITERATIVE REFINED IV METHOD}

The outline of RIV algorithm for the Box-Jenkins model within the closed-loop context (RIVCL) is given below. For ease of notation, the following simplified BJ model with AR noise (as used in the CAPTAIN RIV routine) will be considered here ${ }^{4}$ :

$$
y(t)=\frac{B\left(q^{-1}\right)}{F\left(q^{-1}\right)} u(t)+\frac{1}{D\left(q^{-1}\right)} e(t)
$$

\section{Estimate an ARX model}

Postulate an ARX model

$$
F\left(q^{-1}, \theta\right) y(t)=B\left(q^{-1}, \theta\right) u(t)+e(t)
$$

Apply the LS method

$$
\hat{\theta}^{(0)}=\left[\sum_{i=1}^{N} \varphi(t) \varphi^{T}(t)\right]^{-1} \sum_{i=1}^{N} \varphi(t) y(t)
$$

\footnotetext{
3 http://www.es.lancs.ac.uk/cres/captain/

4 the RIV algorithm in CAPTAIN will shortly be updated to allow for the full BJ model estimation with an ARMA noise model
}

where $\varphi(t)$ is given by (4). This yields $\hat{B}^{(0)}\left(q^{-1}\right)$ and $\hat{F}^{(0)}\left(q^{-1}\right)$. Denote the corresponding transfer function by $\hat{G}^{(0)}(q)$

$$
\hat{G}^{(0)}(q)=\frac{\hat{B}^{(0)}\left(q^{-1}\right)}{\hat{F}^{(0)}\left(q^{-1}\right)}
$$

Set $\hat{D}^{(0)}\left(q^{-1}\right)=1$ and $i=1$.

2. Compute an IV estimate using the estimated prefilter and previous IV estimate for generating the instruments.

Generate the instruments as

$$
\left\{\begin{array}{l}
L_{\text {rivcl }}^{(i-1)}\left(q^{-1}\right)=\frac{\hat{D}^{(i-1)}\left(q^{-1}\right)}{\hat{F}^{(i-1)}\left(q^{-1}\right)} \\
\hat{y}\left(t, \hat{\theta}^{(i-1)}\right)=\frac{\hat{G}^{(i-1)}(q)}{1+C_{c}(q) \hat{G}^{(i-1)}(q)} r(t), \\
\hat{u}\left(t, \hat{\theta}^{(i-1)}\right)=\frac{1}{1+C_{c}(q) \hat{G}^{(i-1)}(q)} r(t), \\
z_{f}(t)=L_{\text {rivcl }}^{(i-1)}(q) \\
{\left[-\hat{y}\left(t-1, \hat{\theta}^{(i-1)}\right) \ldots-\hat{y}\left(t-n, \hat{\theta}^{(i-1)}\right)\right.} \\
\left.\hat{u}\left(t-1, \hat{\theta}^{(i-1)}\right) \ldots \hat{u}\left(t-n, \hat{\theta}^{(i-1)}\right)\right]
\end{array}\right.
$$

$z_{f}(t)$ can be seen as an filtered estimation of the noise-free part of the regressor $\varphi(t)$. Determine the IV estimate using the prefilter and these instruments

$$
\hat{\theta}_{\text {rivcl }}^{(i)}=\left[\sum_{i=1}^{N} z_{f}(t) \varphi_{f}^{T}(t)\right]^{-1} \sum_{i=1}^{N} z_{f}(t) y_{f}(t),
$$

with

$$
\left\{\begin{array}{l}
\varphi_{f}(t)=L_{\text {rivcl }}^{(i-1)}(q) \varphi(t) \\
y_{f}(t)=L_{\text {rivcl }}^{(i-1)}(q) y(t)
\end{array}\right.
$$

This yields $\hat{B}_{\text {rivcl }}^{(i)}\left(q^{-1}\right)$ and $\hat{F}_{\text {rivcl }}^{(i)}\left(q^{-1}\right)$. Denote the corresponding transfer function by $\hat{G}_{\text {rivcl }}^{(i)}(q)$

$$
\hat{G}_{\text {rivcl }}^{(i)}(q)=\frac{\hat{B}_{\text {rivcl }}^{(i)}\left(q^{-1}\right)}{\hat{F}_{\text {rivcl }}^{(i)}\left(q^{-1}\right)}
$$

3. Compute the residuals associated with the RIVCL estimates at iteration $i$ and determine an $A R$ model for these.

Let

$$
\hat{\omega}(t)=\hat{F}_{\text {rivcl }}\left(q^{-1}\right) \tilde{y}_{f}(t)-\hat{B}_{\text {rivcl }}\left(q^{-1}\right) \tilde{u}_{f}(t)
$$

with $\tilde{y}_{f}(t)=1 / \hat{F}_{\text {rivcl }}\left(q^{-1}\right) y(t)$ and $\tilde{u}_{f}(t)=$ $1 / \hat{F}_{\text {rivcl }}\left(q^{-1}\right) u(t)$.

Postulate an AR model for these residuals $\hat{\omega}(t)$ in the form :

$$
D\left(q^{-1}\right) \hat{\omega}(t)=e(t)
$$

Estimate $D\left(q^{-1}\right)$ by LS (or some alternative such as the ARMACEL procedure (Broersen, 2002)) and denote the result by $\hat{D}_{\text {rivcl }}^{(i)}\left(q^{-1}\right)$. 
4. Repeat from step 2. Stop when $\hat{F}_{\text {rivcl }}^{(i)}, \hat{B}_{\text {rivcl }}^{(i)}$ and $\hat{D}_{\text {rivcl }}^{(i)}$ have converged. Considerable previous experience with the RIV algorithm has shown that this iterative relaxation approach normally requires only two to four iterations to converge on a reasonable set of model parameters but a convergence criterion could be incorporated.

\subsection{Comments}

- Adaptive prefiltering of both I/O data signals is an inherent part of the RIVCL estimation.

- This algorithm requires knowledge of the controller but a solution is to estimate the closed-loop TF (instead of the open-loop one) in the first step of the algorithm and then to construct the instruments on the basis of this closed-loop TF and $r(t)$.

\subsection{The Closed-Loop Simplified Refined IV (SRIVCL) algorithm}

Like its close relative RIV, the RIVCL algorithm described above with ARMA or AR noise modelling does not necessarily require the concurrent estimation of a noise model and, indeed, is statistically efficient for this model if the additive noise on the output is white. In the RIV case, this algorithm is called the Simplified RIV (SRIV) algorithm (Young, 1985b) and so we will refer to this closed loop equivalent as the Simplified RIVCL (SRIVCL) algorithm. It is identical to the RIVCL algorithm except that the adaptive prefiltering operation simplifies by setting $D^{(i-1)}\left(q^{-1}\right)=1$.

The SRIVCL algorithm is not only easy to implement since it does not require concurrent estimation of a disturbance model (and is therefore independent of any stochastic assumption about the noise process, which can take on any form), but it also provides very good estimates in many practical situations. Over many years, the SRIV algorithm has proven to be quite robust in the face of non-standard noise on the output signal, producing consistent, asymptotically unbiased and relatively low variance parameter estimates in case of open-loop identification (Young, 1984). The closed loop algorithm is virtually identical to the open loop algorithm and so similar performance is to be expected.

\section{SIMULATION EXAMPLES}

The following numerical example is used to illustrate the performance of the proposed method (Gilson and Van den Hof, 2005). The process to be identified is described by equation (1), where

$$
\begin{aligned}
G_{0}(q) & =\frac{0.5 q^{-1}}{1-0.8 q^{-1}}, \quad n=1 \\
C_{c}(q) & =\frac{0.0012+0.0002 q^{-1}-0.001 q^{-2}}{0.5-0.9656 q^{-1}+0.4656 q^{-2}}
\end{aligned}
$$

$r(t)$ is a deterministic sequence (realization of a random binary signal) and $e_{0}(t)$ is a white noise uncorrelated with $r(t)$.

\subsection{Example 1: white noise}

Firstly, a white noise disturbance $\left(H_{0}(q)=1\right)$ is considered in order to validate the performance of the proposed simplified refined IV (srivcl) algorithm in the case $\mathcal{S} \in \mathcal{M}$. The process parameters are estimated on the basis of closed-loop data sequences of length $N=1000$. A Monte Carlo simulation of 100 experiments has been performed for a signal to noise ratio

$$
S N R=10 \log \left(\frac{P_{y_{d}}}{P_{e}}\right)=15 \mathrm{~dB},
$$

where $P_{x}$ denotes the power of the signal $x$ and $y_{d}$ is the noise-free output signal.

The proposed method is compared to another approximatively optimal IV method referenced as div (without noise model estimation, see Gilson and Van den Hof (2005)). The Monte Carlo simulation (MCS) results are presented in Table 1 where the mean and standard deviation of the estimated parameters are displayed. It can be seen that both methods deliver unbiased and accurate results, with smaller standard errors for the proposed srivcl method.

\begin{tabular}{|c|c|c|c|}
\hline param. & true & div & srivcl \\
\hline$\hat{b}_{1}$ & 0.5 & $0.4999 \pm 0.0062$ & $0.5000 \pm 0.0039$ \\
$\hat{a}_{1}$ & -0.8 & $-0.7996 \pm 0.0033$ & $-0.7999 \pm 0.0023$ \\
\hline
\end{tabular}

Table 1. MCS results $-H_{0}(q)=1$

\subsection{Example 2: colored noise}

A second example is used to analyse the performance of the proposed methods in the case of a colored noise, with

$$
H_{0}(q)=\frac{1-1.56 q^{-1}+1.045 q^{-2}-0.3338 q^{-3}}{1-2.35 q^{-1}+2.09 q^{-2}-0.6675 q^{-3}}
$$

The proposed methods (referred to as rivcl and srivcl) are compared to the div algorithm and another approximatively optimal IV method which estimates an ARARX model and is referred to as divn (Gilson and Van den Hof, 2005). Moreover, note that a simplified BJ noise model is used in the rivcl $\left(C\left(q^{-1}\right)=1\right)$ case. Therefore, the case $G_{0} \in \mathcal{G}$ (and not $\mathcal{S} \in \mathcal{M}$ ) is considered in this example in order to analyse the robustness of the proposed method to the noise.

The process parameters are estimated on the basis 


\begin{tabular}{|c|c|c|c|c|c|}
\hline parameters & true values & div & divn & srivcl & rivcl \\
\hline$\hat{b}_{1}$ & 0.5 & $0.5005 \pm 0.0210$ & $0.5003 \pm 0.0116$ & $0.4998 \pm 0.0212$ & $0.5005 \pm 0.0107$ \\
$\hat{a}_{1}$ & -0.8 & $-0.7995 \pm 0.0352$ & $-0.7993 \pm 0.0139$ & $-0.8001 \pm 0.0120$ & $-0.7997 \pm 0.0080$ \\
\hline
\end{tabular}

Table 2. Mean and standard deviation of the 100 estimated models in the case of example 2

of closed-loop data sequences of length $N=1000$. A Monte Carlo simulation of 100 experiments has been performed for a $S N R=10 \mathrm{~dB}$. The mean and standard deviation of the estimated parameters for the 100 models are given in Table 2. The Bode diagrams of the 100 models identified by the divn and rivcl algorithms are displayed in Figure 2. It can be seen that all of the methods give unbiased and good results. The rivcl and srivcl algorithms seem to give estimates with smaller standard errors compared to the div and divn algorithms.
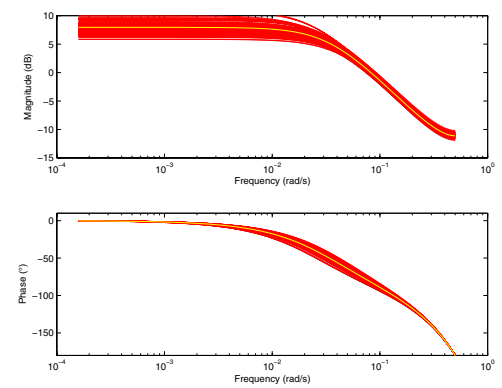

(a) divn
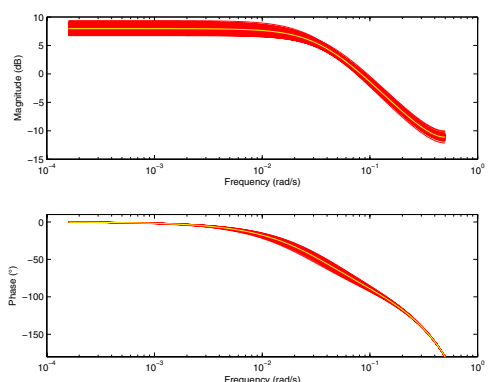

(b) rivcl

Fig. 2. Estimated Bode diagrams over the 100 Monte Carlo simulation runs

\section{CONCLUSION}

This paper has considered the identification of a non-linear-in-the-parameters Box-Jenkins TF model within a closed loop environment, using the optimal Refined Instrumental Variable technique modified to handle the closed loop situation (RIVCL). It has been shown that a minimal value of the associated parametric error covariance matrix can be achieved by the RIVCL choice of instruments and prefilters. The estimated BoxJenkins model has the advantage of not constraining the process and the noise models to have common polynomials. The proposed method has been compared to recently suggested estimators using comprehensive Monte Carlo simulation analysis. All of these methods considered in the paper yield unbiased estimates but the RIVCL algorithm provides the best statistical efficiency.

\section{REFERENCES}

Broersen, P.M.T. (2002). Automatic spectral analysis with time series models. IEEE Transactions on Instrumental Measurement 51(2), 211-216.

Forssell, U. and L. Ljung (1999). Closed-loop identification revisited. Automatica 35(7), 12151241.

Gilson, M. and P. Van den Hof (2001). On the relation between a bias-eliminated least-squares (BELS) and an IV estimator in closed-loop identification. Automatica 37(10), 1593-1600.

Gilson, M. and P. Van den Hof (2005). Instrumental variable methods for closed-loop system identification. Automatica 41(2), 241-249.

Ljung, L. (1999). System identification : theory for the user - Second Edition. Prentice-Hall.

Söderström, T. and P. Stoica (1983). Instrumental variable methods for system identification. Springer-Verlag.

Söderström, T., P. Stoica and E. Trulsson (1987). Instrumental variable methods for closedloop systems. In: 10th IFAC World Congress on Automatic Control. Munich - Germany. pp. 363-368.

Van den Hof, P.M.J. (1998). Closed-loop issues in system identification. Annual Reviews in Control 22, 173-186.

Young, P.C. (1976). Some observations on instrumental variable methods of time-series analysis. International Journal of Control 23(5), 593-612.

Young, P. C. (1970). An instrumental variable method for real-time identification of a noisy process. Automatica 6, 271-287.

Young, P. C. (1984). Recursive estimation and time-series analysis. Springer-Verlag. Berlin.

Young, P. C. (1985a). Recursive identification, estimation and control. In: Handbook of Statistics 5: Time Series in the Time Domain. E. J. Hannan, P. R. Krishnaiah and M. M. Rao (eds.). pp. 213-256.

Young, P. C. (1985b). The instrumental variable method: a practical approach to identification and system parameter estimation In: Identification and System Parameter Estimation. H.A.Barker and P.C.Young (eds). Pergamon Press: Oxford, pp. 1-16 